Hautarzt 2009 - 60:693-694

DOI 10.1007/s00105-009-1799-9

Online publiziert: 12. August 2009

(c) Springer Medizin Verlag 2009

\author{
S.M. John ${ }^{1} \cdot$ M. Jünger ${ }^{2}$ \\ ${ }^{1}$ Abteilung Dermatologie, Umweltmedizin, Gesundheitstheorie, Universität Osnabrück \\ ${ }^{2}$ Klinik und Poliklinik für Hautkrankheiten, Klinikum der \\ Ernst-Moritz-Arndt-Universität Greifswald
}

\title{
Berufsdermatologie 2009
}

\section{Viele neue Optionen für die Praxis}

Anfrage eines Kollegen: „Kann man bei einer Aushilfsjobberin in einer Kfz-Werkstatt mit einem berufskongruenten Handekzem einen Hautarztbericht erstatten?" Antwort: Ja, sollte man! Leider tun das noch nicht alle Kollegen. Dabei können wir unseren Patienten nur so die Umsetzung ihrer Ansprüche an die gesetzliche Unfallversicherung ermöglichen. Ein Frühinterventionsverfahren wie das Hautarztverfahren mit der Möglichkeit, Diagnostik und Therapie zulasten der UVTräger zu erbringen, gibt es von den 68 offiziellen Berufskrankheiten nur bei einer nämlich der „BK Haut“ (BK 5101). Für uns Dermatologen gleichzeitig Privileg und Verpflichtung. Eine Verpflichtung, die wir im Interesse unserer Patienten, aber auch um die Dermatologie als Organfach zu erhalten, annehmen sollten. Die Diagnostik im Hautarztbericht soll berufsbezogen erfolgen; was dabei aktuell allergologisch bei den Hautrisikoberufen zu beachten ist, ist in der konzisen Übersicht des IVDK (J. Geier) zusammengestellt.

\section{? Jüngste Entwicklungen machen es leichter, aktiv Berufsdermatologie zu betreiben.}

Das „optimierte Hautarztverfahren“ und die im aktuellen Ärztevertrag ${ }^{1}$ erstmals festgelegte Verpflichtung der UV-Träger zur „unverzüglichen“ Reaktion auf Hautarztberichte haben das Ziel, die Versorgung von Patienten mit beruflichen Hauterkrankungen in der Praxis noch effizi-

\footnotetext{
$1 \S 41$, Abs. 3, Vertrag Ärzte/UV-Träger vom 1. April 2008.
}

enter durchführen zu können; zudem ist die Honorierung angehoben worden. Und es sind noch weitere wegweisende Entwicklungen der letzten Zeit zu nennen:

- Das verwaltungsseitige Stufenverfahren Haut, mit der Festschreibung gestufter Präventionsmaßnahmen unter Stärkung des ambulanten Heilverfahrens beim Dermatologen sowie des stationär-ambulant vernetzten Heilverfahrens im Sinne der integrierten Versorgung bei schweren Berufsdermatosen; 2 aktuelle Studien analysieren, ob es funktioniert (Beiträge C. Skudlik und H. Voss).

- Flankierende Maßnahmen der Qualitätssicherung durch ABD und DDG: Besonders hervorzuheben ist die gerade publizierte Leitlinie „Management von Handekzemen", die sich mit der Diagnostik, Prävention und aktuellen Therapie von Handekzemen beschäftigt (Beitrag C. Soost und M. Worm); ferner die Leitlinie „Berufliche Hautmittel“, die bereits berücksichtigt ist in den aktuellen Änderungen im einschlägigen Technischen Regelwerk (TRGS 401), das verbindliche Vorgaben für Arbeitgeber in Hautrisikoberufen macht. Weltweit erstmalig wird darin Feuchtarbeit als „Gefahrstoff“ identifiziert (Beitrag M. Fartasch). Ferner sind mit der aktuell publizierten neuen Fassung des Bamberger Merkblattes für die dermatologische Begutachtung Empfehlungen geschaffen worden, die weit über die Begutachtung hinausgehen, insofern als hier erstmals auch berufsbedingter Hautkrebs berücksichtigt wird. In diesem Zusam- 
menhang wird die Tür für die Anerkennung von UV-induziertem beruflichem Hautkrebs geöffnet.

- Die Dermatologie ist durch diese Neuerungen in Zeiten, in denen qualifizierte präventive Angebote zunehmend nachgefragt werden, gut aufgestellt. Zukünftig werden sich die Leistungsstrukturen in Praxis und Klinik noch vermehrt darauf ausrichten. Die $\mathrm{ABD}$ hat in diesem Zusammenhang bereits 600 Kollegen für die Berufsdermatologie zertifiziert; auf die regelmäßigen Zertifizierungsseminare sei hingewiesen; für 2009 ist noch eine Reihe von Übergangsregelungen in Kraft. Ich möchte auch nicht versäumen, Sie herzlich zur 10. ABDTagung (15.-17.10.2009; http:// www. mci-berlin.de/abd2009) nach Osnabrück einzuladen.
„Der Hautarzt" hat zeitnah auf die neuen Entwicklungen reagiert und dieses Themenheft zur Berufsdermatologie ermöglicht; möge es Ihnen als Kompendium und Nachschlagewerk dienen.

\section{Swen Malk John}

Prof. Dr. S.M. John

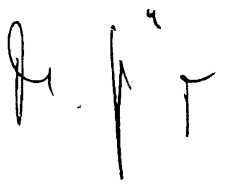

Prof. Dr. M. Jünger

\section{Korrespondenzadressen}

Prof. Dr. S.M. John

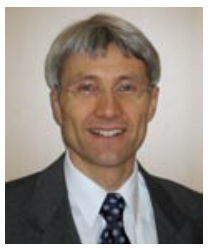

Abteilung Dermatologie, Umweltmedizin, Gesundheitstheorie, Universität Osnabrück Sedanstr. 115 49090 Osnabrück sjohn@uos.de

\section{Prof. Dr. M. Jünger}

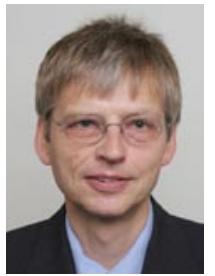

Klinik und Poliklinik für Hautkrankheiten, Klinikum der Ernst-Moritz-ArndtUniversität Greifswald Fleischmannstr. 42-44 17487 Greifswald juenger@uni-greifswald.de

\section{(7) Exklusiv für Abonnenten:}

\section{Nutzen Sie das Online-Archiv von Der Hautarzt}

Ihre Vorteile:

- Komfortable und schnelle Recherche nach Themen, Autoren, Suchbegriffen

- Ob unterwegs oder am eigenen PC: Zugriff überall und jederzeit

— Online First: Lesen Sie die aktuellsten Beiträge schon vor Erscheinen des gedruckten Heftes online

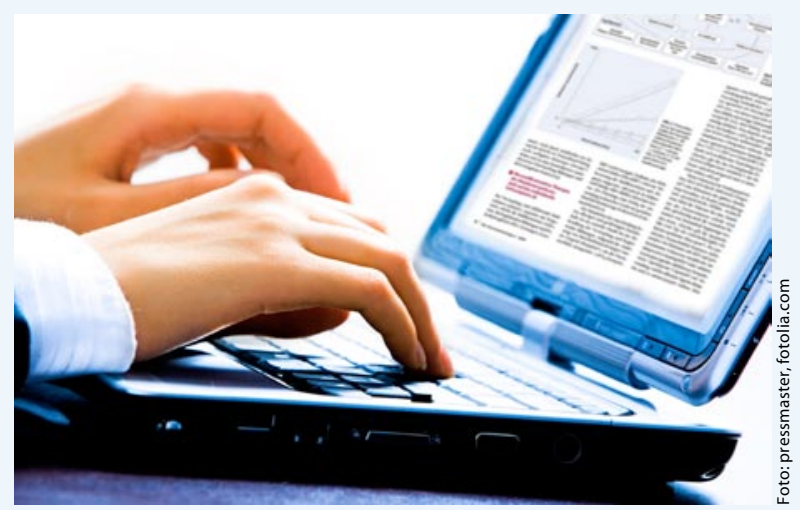

